Questions vives

\section{Questions Vives}

Recherches en éducation

$\mathrm{N}^{\circ} 33 \mid 2020$

Visées heuristiques, praxéologiques et critiques dans les recherches participatives en éducation

\title{
L'adossement réflexif à la recherche comme activité heuristique, praxéologique, critique
}

Reflexive backing to research as heuristic, praxeological and critical activity

\section{Nicolas Sembel}

\section{(2) OpenEdition}

\section{Journals}

Édition électronique

URL : http://journals.openedition.org/questionsvives/4539

DOI : 10.4000 /questionsvives. 4539

ISSN : 1775-433X

Éditeur

Université Aix-Marseille (AMU)

Édition imprimée

Date de publication : 15 décembre 2020

ISBN : 978-2-912643-57-5

ISSN : $1635-4079$

\section{Référence électronique}

Nicolas Sembel, «L'adossement réflexif à la recherche comme activité heuristique, praxéologique, critique », Questions Vives [En ligne], №33 | 2020, mis en ligne le, consulté le 25 mars 2021. URL http://journals.openedition.org/questionsvives/4539; DOI : https://doi.org/10.4000/questionsvives. 4539

Ce document a été généré automatiquement le 25 mars 2021.

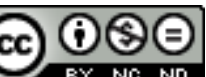

Questions Vives est mis à disposition selon les termes de la licence Creative Commons Attribution Pas d'Utilisation Commerciale - Pas de Modification 4.0 International. 


\section{L'adossement réflexif à la recherche comme activité heuristique, praxéologique, critique}

Reflexive backing to research as heuristic, praxeological and critical activity

Nicolas Sembel

\section{Introduction : des perspectives nouvelles}

1 Quelles perspectives scientifiques s'ouvriraient avec la systématisation du recoupement des trois visées heuristique, praxéologique et critique proposées par le symposium, comme critère unique d'opérationnalisation de la recherche? En quoi le fait de définir chacune des trois visées essentiellement par son recoupement avec les deux autres (par exemple, ne définir l'heuristicité qu'en la croisant avec la praxéologie et la critique, etc.) permettrait-il de revisiter le projet épistémologique et les pratiques de recherche des sciences de l'éducation? Notre hypothèse principale est que ce recoupement des trois visées s'intensifierait encore s'il était traversé par la réflexivité, définie comme autosocioanalyse (Bourdieu, 2001, 2003b). La réflexivité pourrait devenir le dénominateur commun à l'heuristique, au praxéologique et au critique; comment redéfinirait-elle l'activité intellectuelle, la posture épistémologique, les pratiques scientifiques associées à ces trois visées ? Dans cet article, nous testerons cette méthode de croisement «dense » (Geertz, 1998), de "condensation» des trois visées, sur un objet d'étude qui reste largement à construire : l'adossement réflexif à la recherche, à travers l'exemple de l'adossement de la formation à la recherche en Inspé (Institut national supérieur du professorat et de l'éducation). La dimension réflexive de cet adossement nous intéresse particulièrement depuis notre arrivée en 2016 à l'Inspé d'Aix-Marseille Université, en tant que sociologue dans une équipe de recherche en ergonomie des professions enseignantes (ex-Ergape, aujourd'hui Claef, Clinique des activités en éducation et en formation, laboratoire Adef). Notre credo est le suivant : les conditions extrêmes offertes par le contexte scolaire marseillais permettront, si elles 
sont exploitées à bon escient, de retrouver le projet républicain révolutionnaire centré sur l'esprit critique, l'instruction, l'élémentation, la science.

2 Faute de texte officiel explicatif et de tradition théorique notoire, la notion d'adossement peut être définie par son étymologie, par exemple l'adossement d'une véranda contre une façade : adosser à la recherche signifie que celle-ci est l'élément principal, et que les éléments adossés s'appuient sur elle, de façon harmonieuse, pour que l'ensemble soit agréable et fonctionnel. L'harmonie architecturale répond à des contraintes techniques, mais surtout à un choix de vie comme "toile de fond", qui donne une direction, un esprit, ou, dans le cas qui nous intéresse, une imagination scientifique. La traduction anglaise par "backing", qui fait écho à "background", traduit cette idée. Un synonyme possible d'adosser est arrimer (à la recherche); et un antonyme, séparer (distinguer). Adosser systématiquement à la recherche chaque heure de formation, initiale et continue, de tous les publics, et chaque heure d'apprentissage, depuis l'école maternelle, de tous les élèves, aurait quelles conséquences heuristiques, praxéologiques et critiques sur l'activité intellectuelle au sens le plus large du terme, sur le rapport à la recherche au sens large, sur le rapport au savoir, « tout au long de la vie»?

3 Nous décrirons successivement cet adossement sur les plans épistémologique, théorique, didactique, réglementaire, empirique. Ces cinq points sont imbriqués : les fondements épistémologiques réflexifs orientent vers un rapport sociogénétique à la théorie, conditionnant la généralisation de la démarche de didactique de l'enquête, confortée par une lecture réglementaire « condensée », justifiant l'approche empirique, notamment clinique, de la recherche collaborative décrite à la fin. Autrement dit : une posture réflexive ancre le rapport aux éléments théoriques et aux prescriptions officielles dans une démarche d'enquête empirique collaborative.

\section{Les conditions épistémologiques de l'adossement : du formel au réflexif}

4 Nous voudrions d'abord défendre, en tant que chercheur, la proposition selon laquelle l'épistémologie n'a de sens que parce qu'elle résout les problèmes pratiques posés par la recherche, et qu'elle est la seule à pouvoir les résoudre. S'il y a donc bien une nécessité épistémologique, si l'épistémologie a inévitablement des aspects formels, il y a aussi une posture épistémologique. Laquelle ne peut être qu'ancrée dans la pratique, qui seule peut constituer une ressource pour dynamiser l'activité intellectuelle, et agir contre l'empêchement de celle-ci. Posture, pratique et activité ouvrent toutes vers une réflexivité objectivée, et son possible développement vers une objectivation participante (Bourdieu, 2003a) généralisée ( $c f$. point 5). La posture, nous le verrons, n'est pas liée à un statut particulier, ni l'activité à un contexte précis; en ce sens, la démarche épistémologique pour laquelle nous plaidons a une portée universaliste, comme la science qu'elle anime en pratique, qu'elle tend à rendre vivante, qu'elle cherche à protéger des "savoirs moribonds » (Chevallard, 1998). Le croisement des trois visées, heuristique, praxéologique et critique, donne à cette perspective épistémologique sa pleine dimension, et ouvre vers la possibilité de revisiter l'entreprise épistémologique des sciences de l'éducation. En particulier, elle permet de revisiter aussi le " triptyque » très lié aux sciences de l'éducation et qui est au cœur des Inspé, en cherchant à relier, toujours un peu plus, la formation des enseignants, leurs 
enseignements, l'apprentissage de leurs élèves. Soit trois activités intellectuelles toutes potentiellement adossables à la recherche, trois postures de chercheurs qui, par-delà leurs différences, sont potentiellement inscrites dans une démarche scientifique, et donc universelle, d'enquête.

Nous postulons comme hypothèse de travail que les trois visées sont inséparables, et non hiérarchisables. Une praxéologie sans prise en compte de l'heuristicité n'est qu'illusion de scientificité, une expertise sans science car ne posant que les questions heuristiques qui auraient une réponse praxéologique. Une heuristicité sans objectif praxéologique n'est qu'illusion de sciences humaines et sociales, sciences aussi scientifiques que les autres de par leur démarche et leur quête de vérité, mais différentes de par leurs objets, souvent des questions socialement vives (Legardez et Simmoneaux, 2006), leurs terrains, et leurs méthodes, impliquant l'humain. Enfin, il n'y a pas d'heuristicité qui ne critique la praxéologie, lorsque celle-ci prétend échapper à la science; pas de praxéologie qui ne critique l'heuristicité, lorsque celle-ci prétend échapper au réel, au social, au vivant; pas de critique sans heuristicité, qui ne serait que militante ; pas de critique sans praxéologie, qui la rend constructive.

Un dernier élément important de définition reste à préciser, last but not least, pour parachever l'ancrage de notre argumentation épistémologique dans la pratique: la dimension réflexive. Définie dans notre perspective comme une compétence intellectuelle à se distancier par autosocioanalyse, elle ne constitue pas une quatrième visée mais est selon nous indissociable des trois autres, qu'elle permet de revisiter. Elle est décisive pour leur développement et leur articulation : elle est en effet au cœur des pratiques, des postures, des activités. Elle oriente le rapport de l'activité intellectuelle au savoir, à la recherche, à l'opérationnalisation et au déploiement de cette dernière. Que seraient l'heuristicité, la praxéologie, la critique, sans la réflexivité ? L'heuristique intègre notre propre rapport à la recherche, la critique notre propre position, la praxéologie notre propre pratique. La réflexivité intellectuelle est le garant du vivant, de la découverte, de l'imagination scientifique, de l'objectivation participante; le garde-fou contre le moribond, le critère d'une épistémologie ancrée. L'adossement réflexif à la recherche est un produit du croisement entre les trois visées, heuristique, praxéologique et critique. En transposant le plus heuristiquement possible la recherche dans tous les domaines de l'activité intellectuelle, aucun aspect de cette activité n'échappe potentiellement à cette praxéologie généralisée. Et d'autres aspects se trouvent disqualifiés par la critique systématique de tout ce qui ne s'inscrit pas dans cette heuristicité et cette praxéologie. Enfin, l'adossement est réflexif de par la place et le rôle centraux qu'il joue dans le développement de toute activité intellectuelle.

\section{Les conditions théoriques de l'adossement : d'un rapport académique à un rapport sociogénétique aux savoirs}

7 La combinaison des visées heuristique, praxéologique et critique fonctionne particulièrement bien pour construire en pratique les bases théoriques de l'adossement à la recherche, en les revisitant par l'épistémologie redéfinie dans le sens que nous venons de proposer. Contre toute théorisation coupée de son contexte de production, il s'agit au contraire de cerner systématiquement les fondements pratiques de toute 
théorisation, c'est-à-dire la sociogénèse de tout concept (Heilbron, 2018). Ainsi relus, des sociologues « classiques » des sciences de l'éducation comme Durkheim et Bourdieu (sur lesquels nous travaillons), trouvent une actualité nouvelle, particulièrement pour l'analyse, la compréhension et la recontextualisation de leur activité intellectuelle. L'heuristicité de leurs argumentations scientifiques a fondé leur réputation ; il convient néanmoins de revisiter ces argumentations en les croisant avec une praxéologie, qu'ils ont toujours appelée de leurs vœux, une critique, dont ils sont d'infatigables animateurs, et leur réflexivité, introduite dans l'analyse par des recoupements avec des éléments habituellement classés comme des "éclairages biographiques ». Si l'on pousse cette logique jusqu'au bout, il est possible de ne considérer comme véritablement intéressantes que les productions scientifiques de tout chercheur relues en lien avec son activité intellectuelle et réflexive, ce que Waquet appelle l'ordre matériel du savoir (Waquet, 2015). Egalement, cette démarche remet de facto en cause les cloisonnements non seulement disciplinaires, mais aussi scientifiques, que l'activité réelle est amenée à déborder. Elle ouvre ainsi un espace pour une définition opératoire des sciences de l'éducation scientifiquement plus heuristique que les définitions, souvent empilées et juxtaposées, de toute science au singulier. Et, bien que la philosophie ne se définisse pas comme une démarche scientifique, certains argumentaires philosophiques sont parfois plus heuristiques, lorsqu'ils parviennent eux aussi au croisement dense des trois visées, que certains argumentaires des sciences humaines et sociales qui "densifieraient" moins. Car au final, ne sont pertinentes, dans la perspective que nous défendons, que les productions et démarches à la fois heuristiques, praxéologiques et critiques, toutes en lien avec leur dimension réflexive intrinsèque de l'activité intellectuelle qui leur a donné naissance. Nous avons conscience que, dans un premier temps et dans les limites de cet article, nous ne donnons pas la place que mériterait, dans un écrit plus conventionnel, la tradition théorique d'analyse distinguant soigneusement, dans un premier temps, l'heuristicité, la praxéologie et la critique, pour examiner dans un second temps les combinaisons possibles entre ces trois concepts.

8 La sociologie, les sciences de l'éducation, ne sont plus alors des disciplines en quête de cloisonnements avec d'autres disciplines, ni de cloisonnements entre des courants, écoles, mentors, concepts, méthodes, etc.; mais inscrites dans une démarche scientifique, complémentaire avec d'autres, avec toutes les autres. La puissance de cette démarche, qui ne se perd pas dans des impasses théoriques, par exemple celle des «ismes» (Boschetti, 2014), s'enrichit du croisement avec le souci heuristique, l'exigence praxéologique et la dynamique critique, qui la renforcent, et le sousbassement réflexif, qui la spécifie. Chaque aspect de la pensée de Durkheim et de Bourdieu (pour en revenir à eux) sur l'école peut être restitué avec toute sa part d'heuristicité, de praxéologie et de critique, à laquelle leurs réflexivités respectives donnent tout son sens. Il n'est plus possible alors de mettre de côté le rapport réflexif de Durkheim et de Bourdieu à l'école, respectivement juif et béarnais, dominés puis dominants, etc., source de leur critique de l'école. Ils ne peuvent penser scientifiquement, et nous ne pouvons comprendre leur geste scientifique, sans intégrer cette réflexivité et cette critique, et sans imaginer développer aucun argument qui n'ait pas de conséquences heuristiques et praxéologiques. Il est ainsi possible de reconstituer la « sociogénèse » forcément réflexive, les «fondements pratiques » de leurs concepts, ce qui laisse percevoir leurs inséparables heuristicité, critique et praxéologie. Par exemple, quand Durkheim considère que l' " effervescence » de la vie collective de la classe, qui n'est pas une "foule", peut être "salutaire " (Durkheim, 1925/1992), il 
procède à une analogie physiologique, qui ne réduit pas sa description de la classe à un organisme humain et ne fait pas de lui un "organiciste", mais qui, au contraire, montre qu'il existe, pour le maître, un levier puissant pour utiliser l'énergie des élèves au profit de leurs apprentissages, analogue à celle qui donne lieu à des manifestations d'extase religieuse ou politique, symptômes de conscience collective. Durkheim investit donc son rapport à la religion, son rapport à l'école, son rapport à la science, pour dénicher la clé de l'analyse de l'activité intellectuelle des enseignants et des élèves, critiquer ce qu'elle n'est pas, puis en faire un objet de formation dans ses cours de science de l'éducation et de recherche dans ses écrits. Tout chez lui est intégré autour d'une conception universelle de cette activité chez les élèves, les enseignants, les formateurs, les savants. Cette activité est une démarche d'enquête, qui fait "sortir » chacun de lui-même, lui fait découvrir et analyser le monde social et sa relation à ce monde. Autre exemple, Bourdieu refuse de définir académiquement la «violence symbolique ", et passe sa vie intellectuelle, avec puis sans Passeron, à la faire vivre empiriquement, sur la base de l'objectivation du consentement intellectuel des élèves, parents, étudiants de catégories populaires "dominés " par cette violence dans le monde scolaire et universitaire. Il investit dans sa démarche un souci permanent d'heuristicité (tester inlassablement sur tous les terrains l'universalité de la pertinence du concept), de praxéologie (chercher sans relâche une alternative pédagogique et didactique à ce «modèle»), de critique (alimenter sans concession et sans fin le dévoilement de tout ce qui légitime ou refoule l'existence de cette violence). Son souci est permanent, inlassable, sans relâche et sans concession car ancré dans sa réflexivité.

Dans cette perspective, il n'est alors plus possible de faire un usage morcelé, édulcoré, théoricisé, académisé, des raisonnements scientifiques de Durkheim et de Bourdieu produits par leur activité intellectuelle ainsi prise en compte. Il est alors possible de comprendre qu'eux-mêmes ne veulent laisser aucun petit Durkheim ou petit Bourdieu être écarté, par l'école, par leurs maîtres, par personne, de la "chaleur de la science " (Durkheim, 1883/1975). Ils font le pari d'un développement possible de l'activité intellectuelle dès la prime enfance et tout au long de la vie, dans le cadre de l'école et de la société républicaines. Et il est symptomatique de constater qu'ils relèvent ce pari en prônant, pour tous, dans le cadre ouvert d'une démarche que nous avons appelée de " sociologie générale de l'éducation », la « sortie de soi » (Durkheim, 1938/1990, op.cit.), l'«autosocioanalyse» (Bourdieu, op. cit.). Ce sont deux processus continués de développement intellectuel, et de démarche de recherche définie par son plus petit commun dénominateur: la démarche d'enquête. Durkheim trouve sa base dans la promotion « révolutionnaire » du " germinal » par Lakanal et son développement chez Dewey (cf. Dewey, 1993 ; Thiévenaz, 2019), le pédagogue dont il était le plus proche avec Rabelais et Coménius. Bourdieu en a fait le plaidoyer dans son rapport co-écrit avec Gros, et Lahire l'a prolongé par son credo en faveur des sciences du monde social dès l'école primaire. Chevallard (1997), Ladage et Chevallard (2011), Thiévenaz (2019) en ont présenté les fondements didactiques.

\section{Les conditions didactiques de l'adossement : des disciplines à l'enquête}

10 Le principe est le suivant. Contre la conception d'une culture comme fin en soi, et segmentée en millefeuille ou en programme, en listes de réponses, qui fondent par 
exemple le "bluff» de la culture générale (Bourdieu), forme suprême du savoir mort, ou du moins «moribond", s'oppose une diversité de contenus "triés » par l'intérêt intellectuel, appréhendés sous forme de listes de questions, conception d'une culture comme point de départ, actualisant l'idéal révolutionnaire de l'instruction par esprit critique (Condorcet) et élémentation (Lakanal) (Sembel, 2003, 79-80). «Resserrer un long ouvrage, c'est l'abréger, alors qu'en présenter les premiers germes, et à travers eux la matrice, c'est l'élémenter » (Lakanal, 1795, 7). La matrice, c'est la recherche ; l'élémentation, l'adossement à la recherche ; les germes, le questionnement. « Abréger le savoir réduit la culture commune à un minimum basique. On sélectionne les informations par soustraction, en éliminant celles qui paraissent trop complexes [...]. Elémenter le savoir fait envisager la culture commune comme un tremplin. Elle n'est plus définie par soustraction, mais par distillation » (Astolfi, 2008).

11 Par conséquent, avec l'élémentation, un rapport scientifique, épistémologique, didactique et critique aux contenus et aux disciplines se substitue au rapport scolaire historiquement producteur d'abrègements, rapport notamment monovalent, bivalent, polyvalent, encyclopédiste, éclectique, formaliste, hiérarchisé, discriminatoire, aux contenus et aux disciplines. L'accumulation maximale et superficielle des savoirs, source de bluff, concours compris, cède la place à leur diversification maximale et adossée, source de reconnaissance (Abdallah-Pretceille, 1999). Mais dans l'école d'aujourd'hui, la raison scolaire l'emporte trop souvent sur la raison scientifique. Contre cette tendance, les contenus peuvent être articulés selon une démarche de recherche, qui montre leur construction sociale et oblige à leur problématisation et à leur opérationnalisation; les disciplines peuvent être articulées dans un contexte épistémologique, qui les réinscrit dans leur histoire, leur culture, et donc dans la relativité de leur démarcation et de leur identité. L'adossement à la recherche réinscrit la connaissance dans le social et renforce le rapport scientifique, et non scolaire, aux savoirs, aux thématiques et aux disciplines. L'intérêt intellectuel redevient le critère principal d'organisation de l'activité et du rapport aux savoirs, en poussant chez chacun le rapport à la discipline vers la recherche, son adossement, son acculturation, son épistémologie, et enfin sa didactisation scientifique.

La liste des "bases ", "attendus ", «savoirs fondamentaux ", «nombre d'heures de cours sur... », etc., disparaît dans sa primauté, son rudimentaire, sa "pédagogie du simple» (Ferry, critiqué par B. Charlot), sa sacralisation parfois, et son monopole. Elle cède la place à une liste des micro-compétences de l'adossement à la recherche, " habitudes intellectuelles de l'esprit d'enquête » (Lahire, 2007), " gestes de l'enquête » (Ladage \& Chevallard, 2011) de chercheurs, d'enseignants, d'élèves. Compétences liées à l'activité, pas nouvelles en soi mais nouvellement structurantes du fait de leur retour au premier plan, contre le formalisme disciplinaire actuel, qui prend toutefois une place dominante dans une histoire millénaire (Durkheim, 1938/1990). Pêle-mêle: diagnostiquer, décontextualiser, distancier, recontextualiser, déconstruire, élémenter, analogiser, génériciser, questionner, problématiser, déspécifier, observer, analyser, évaluer, critiquer, raisonner, didactiser, "débrouiller» (Lévi-Strauss), etc. Puis, des objets sont à (re)construire, par le croisement de regards pluri-catégoriels réunis dans une même démarche scientifique : l'adossement à la recherche, le milieu de travail, l'établissement formateur, l'articulation recherche-formation-terrain, la réflexivité intellectuelle, etc. Le tout à partir de "quelques pistes telles la sélection d'études de cas prototypiques et diversifiés, la construction de principes d'analyse et de principes 
d'opérationnalisation des contenus et des situations, la formation aux ressources et aux pratiques d'auto-formation..." (Reuter, in Lebeaume et al., 2007). "Sans chercher à combler de supposés "trous" de culture ", et dans une perspective du développement, problématisé avec tous et en situation, du «curriculum potentiel » (Martinand, ibid.) contenu en germe dans la zone proximale de développement intellectuel de chacun. Il s'agit d'une révolution dans le rapport au savoir et dans le rapport à la recherche, qui passe par un adossement généralisé des savoirs, des disciplines, des formations, des enseignements et des apprentissages, à la recherche.

13 Trois exemples convergents de possible adossement à la recherche, issus de la formation des enseignants la plus récente, permettront de faire le lien avec le point suivant. Exemple 1, toutes les "éducations à » sont légitimes, à condition qu'elles s'inscrivent dans une logique (et une compétence) de déspécification en vue d'une éducation inclusive universelle. Exemple 2, les «élèves à besoins éducatifs particuliers " sont surtout révélateurs de la nécessaire généricité de leur intégration, qui passe par la disparition à terme de leurs dispositifs spécifiques de prise en charge, dans un contexte devenu véritablement « ordinaire» d'intégration de toute diversité. Exemple 3, l'éducation au développement durable est devenue l'éducation à la démarche au développement durable : l'expertise recherchée ne se situe plus dans des objets (tel domaine, tel territoire...), mais dans une démarche, analogique et comparative, potentiellement adossable à la recherche. Dans les trois cas, chaque exemple mobilisé ne renvoie pas à un savoir disciplinaire mais à une étape de recherche, et devient un « cas particulier bien construit » sur les plans heuristique, praxéologique et critique, ou encore un savoir scientifique élémenté ouvrant sur un universalisme promu, dans les trois cas, par une démarche d'inclusion.

\section{Les conditions réglementaires de l'adossement : du disciplinaire au « commun »}

14 Les textes produits ou actualisés dans le sillage de la Loi d'orientation et de programmation pour la refondation de l'école de la République du 8 juillet 2013 installent formellement l'adossement à la recherche. «La recherche sera au cœur des enseignements qui seront dispensés au sein des Espé » (Loi de 2013). "Les formations dispensées s'inscrivent dans les cadres disciplinaires et de la recherche [...], notamment par l'adossement à la recherche " (Arrêté du 28 mai 2019 modifiant celui du 27 août 2013 fixant le cadre national des formations dispensées au sein des masters Meef, article 2; l'article 3 s'appuie sur le référentiel des compétences, l'article 4 sur l'alternance intégrative, les annexes précisent les modalités du dispositif d'adossement, et donnent à voir tout son potentiel). "Les stages donnent lieu à un temps de préparation, une phase d'accompagnement [...] et une phase d'exploitation et d'analyse réflexive" (Ibid., article 11). "S'appuyer sur la recherche: indispensable pour construire et fonder la formation, mais aussi pour créer les possibilités de sujets de mémoire qu'elle offre aux étudiants et enseignants-stagiaires et pour développer l'acquisition d'une posture réflexive»; "cet adossement à la recherche vaut pour toutes les formations" (Arrêté du 22 janvier 2014 fixant le cadre national des formations conduisant à la délivrance des diplômes nationaux [...] de master, article 17), et ouvre la voie à de possibles poursuite d'études en doctorat. Les concours de recrutement sont impactés par cette politique : la note des trois directions du Ministère 
de novembre 2019 précise le principe du développement possible de l'adossement tel que nous le définissons dès les épreuves écrites d'admissibilité, et non plus seulement avec l'épreuve d'oral professionnel d'admission. La seconde épreuve écrite des concours précise en effet l'importance de «l'approche critique» comme constitutive $\mathrm{du}$ métier d'enseignant et de CPE, pour développer «l'analyse critique " dans les apprentissages, en conformité avec l'article 15 de la loi de 2013, qui reconnaît dans sa section 4 consacrée au socle commun des connaissances, des compétences et de la culture, « l'esprit critique » des élèves.

Le « commun " généralisé, comme objet de formation adossée à la recherche, est au cœur de cette nouvelle impulsion politique, avec le Tronc commun (TC) de formation des enseignants et des CPE (Note des services centraux du Ministère de l'Education nationale du 30 avril 2015); le référentiel des compétences communes (RCC) des enseignants et des CPE (Bulletins Officiels du 25 juillet 2013 et du 26 mars 2015); et le Socle commun (SC) des connaissances, des compétences et de la culture (décret du 31 mars 2015). Mais l'adossement du commun à la recherche est loin d'être réalisé, accepté, et même compris.

La longue liste officielle des «sous-domaines» tous légitimes et intéressants du TC énumérés dans la note ministérielle a pu donner lieu à une organisation juxtaposée de sous-thématiques compliquant l'adossement à une démarche dynamique de recherche. En enfermant le formateur intervenant au niveau master dans un rapport scolaire à son savoir, conçu en termes de "viatique» (J. Ferry), de «kit de survie», de "smic culturel ", sans définition du "commun" ni de l'adossement à la recherche mais bénéficiant néanmoins de ses éclairages scolarisés (une accumulation de « minimums à savoir sur", listes de dates, d'auteurs, d'œuvres, de définitions, de concepts, de thématiques, de projets, etc.), cette organisation enferme aussi le public en formation, et les futurs apprentissages de leurs élèves, dans le même rapport au savoir. Et ce, en mobilisant des compétences empêchant également l'adossement à la recherche: rapport abstrait à la théorie, abordée comme un catalogue de concepts, d'auteurs, de courants, d'écoles; veille documentaire réduite à des compétences techniques et non adossée à la recherche, exclusion de toute problématisation critique, de toute hypothèse « hardie ", de tout recueil rigoureux de matériau lors de stage d'observation, de toute production scientifique de données, de toute analyse critique, etc. La formation commune reste cependant un enjeu disputé au sein des institutions de formation des enseignants et des CPE. L'évolution à venir dans les nouvelles maquettes peut accentuer encore la place du commun via des "ilôts de culture commune " disséminés, tels des chevaux de Troie, dans les parcours "disciplinaires». La métaphore de la ruse polémologique nous semble à peine exagérée, tant l'objectif est explicitement de révolutionner le rapport au concours. Ce rapport disciplinaire aux savoirs dans le second degré et pluridisciplinaire dans le premier degré évoluerait résolument dans le sens d'une analyse scientifique du métier en contexte, et du développement de l'esprit critique à tous les étages. Et ceci, grâce à un adossement réflexif à cet objet de recherche complexe et passionnant, revisité par la nouvelle politique : les « savoirs pour le métier d'enseignant » (SME).

17 Même difficulté à percevoir l'adossement à la recherche dans les 2 textes sur le RCC. Il faut aller jusqu'au bout de la lecture des 14 compétences communes référencées, car seule la dernière, la CC (compétence commune) $n^{\circ} 14:$ : S'engager dans une démarche individuelle et collective de développement professionnel ", fait spécifiquement et 
explicitement référence à l'adossement à la recherche. Elle se décline en 4 points prometteurs : 1) "compléter et actualiser ses connaissances scientifiques, didactiques et pédagogiques »; 2) «se tenir informé[e] des acquis de la recherche afin de pouvoir s'engager dans des projets et des démarches d'innovation pédagogique visant à l'amélioration des pratiques "; 3) « réfléchir sur sa pratique - seul[e] et entre pairs - et réinvestir les résultats de sa réflexion dans l'action »; et 4) "identifier ses besoins de formation et mettre en œuvre les moyens de développer ses compétences [souligné par nous] en utilisant les ressources disponibles ». Mais la révolution dans l'organisation de la formation universitaire qu'elle provoquerait si elle devenait une "compétence-moteur", autre cheval de Troie, explique son confinement en Inspé, quand elle y est identifiée et promue, ce qui n'est pas toujours le cas; de ce fait, elle a peut-être plus de potentiel d'opérationnalisation délocalisée en établissement formateur (cf. point suivant).

Enfin, si le troisième texte, le SC version 2015, est aujourd'hui un document qui oriente l'activité intellectuelle de l'élève vers l'adossement à la recherche, il a fallu rompre avec les versions antérieures centrées sur les disciplines et étrangères à une telle orientation. Pour rappel, le SC promu par Thélot en 2004, actualisant le « simple» de Ferry cent-vingt ans après et le « smic » de Haby trente ans après, se limite au français, aux mathématiques, à l'anglais commercial, et à quelques notions d'informatique. La Loi de 2013 rompt avec cette logique millénariste d'appauvrissement culturel (Durkheim, 1938/1990, op. cit.), et institutionnalise pour la première fois la notion d'« esprit critique» (art. 15, op. cit. ), à développer chez les élèves. Ce qui suppose que cet esprit soit également développé chez les enseignants et les CPE, ces derniers pouvant être, de ce point de vue, définis comme des « experts critiques » de sociologie et philosophie appliquées, et de mise en pratique du droit - toutes spécialités adossables à la recherche. « Le socle commun doit devenir une référence centrale pour le travail des enseignants et des acteurs du système éducatif ». Troisième " pilier » du commun, le SC « ouvre à la connaissance, forme le jugement et l'esprit critique, à partir d'éléments ordonnés de connaissance rationnelle du monde", "accompagne et favorise le développement physique, cognitif et sensible des élèves", leur permet de " conquérir leur autonomie ». Pour ce faire, l'élève « apprend à réfléchir, à mobiliser des connaissances, à choisir des démarches et des procédures adaptées, pour penser, résoudre un problème, réaliser une tâche complexe ou un projet ». De leur côté, «les enseignants définissent les modalités les plus pertinentes pour parvenir à ces objectifs en suscitant l'intérêt des élèves [posé par la Loi de 2013 comme un "droit » et non plus comme une capacité, par définition inégalement répartie], et centrent leurs activités ainsi que les pratiques des enfants et des adolescents sur de véritables enjeux intellectuels".

Dans ce sens, une définition selon nous heuristique, praxéologique et critique de la compétence est proposée : «Une compétence est l'aptitude à mobiliser ses ressources (connaissances, capacités, attitudes) pour accomplir une tâche ou faire face à une situation complexes ou inédites [souligné par nous]. Compétences et connaissances ne sont ainsi pas en opposition ». Cette définition ambitieuse de la compétence permet d'invalider de facto nombre de pseudo-compétences, à la fois pas assez transposables, pas assez contextualisées, pas assez discriminantes, pas à la hauteur de la définition. Adossée aux tâches et à la dynamique de la recherche, la compétence est l'aptitude pour les élèves comme pour les enseignants et les formateurs (et les chercheurs...) à mobiliser de façon critique des ressources scientifiques, à mettre en œuvre «la 
réflexion critique, la créativité, [...] la résolution de problèmes ». Le SC s'organise en cinq domaines de connaissance, qui brisent les cloisonnements disciplinaires et scientifiques, et sont organisés autour d'une démarche de questionnement. Par exemple, domaine 2 : les «méthodes et outils pour apprendre " amènent à « résoudre un problème, comprendre un document, rédiger un texte, prendre des notes, effectuer une prestation ou produire des objets", de façon méthodique. C'est surtout dans le domaine 4 , « les systèmes naturels et les systèmes techniques ", que l'adossement à la recherche comme démarche scientifique est ici le plus précisément décrit. Pour l'élève, « il s'agit d'éveiller sa curiosité, son envie de se poser des questions, de chercher des réponses et d'inventer ». Et ce qui est dit des élèves est supposé maîtrisé par les enseignants et les CPE : «L'élève sait mener une démarche d'investigation. Pour cela, il décrit et questionne ses observations ; il prélève, organise et traite l'information utile ; il formule des hypothèses, les teste et les éprouve; il manipule, explore plusieurs pistes, procède par essais et erreurs; il modélise pour représenter une situation; il analyse, argumente, mène différents types de raisonnements (par analogie, déduction logique...) ; il rend compte de sa démarche. Il exploite et communique les résultats de mesures ou de recherches en utilisant les langages scientifiques à bon escient ». Il « imagine, conçoit et fabrique des objets et des systèmes techniques. Il met en œuvre observation, imagination, créativité, sens de l'esthétique et de la qualité, talent et habiletés manuels, sens pratique, et sollicite les savoirs et compétences scientifiques, technologiques et artistiques pertinents ".

Replacé par les textes officiels au cœur de l'activité intellectuelle des enseignants, des $\mathrm{CPE}$, et des élèves, le commun que nous venons de condenser est un bel exemple de croisement des visées heuristique, praxéologique et critique, autour d'un adossement réflexif à la recherche. Nous allons terminer par le lieu formateur idéal selon nous, car contextualisé : l'école ou l'établissement particulièrement en REP+ (mais pas que). Alors que les Inspé, parfois drapés dans le «splendide isolement » des prestigieux bâtiments des anciennes Ecoles normales, sont amenés à «délocaliser » (par exemple avec les Travaux dirigés délocalisés, TDD), comment penser une formation « relocalisée »?

\section{Les conditions empiriques de l'adossement : de l'éclairage à la clinique}

21 Empiriquement, Durkheim et Bourdieu se rejoignent pour défendre une posture de recherche empirique fondée sur la «sortie de soi» (Durkheim, 1938/1990, op. cit.), synonyme de "réflexivité » (Bourdieu, 2003b, op. cit.), devenant au contact avec le «terrain » une "objectivation participante » (Bourdieu, 2003a, op. cit.), laquelle fait directement écho aux trois visées d'heuristicité, de praxéologie et de critique. La pratique est ici source d'heuristicité et garantie de critique. Selon notre lecture, cette objectivation participante généralisée à tous les protagonistes permet de renforcer la recherche collaborative.

La recherche collaborative (forme de recherche participative), est directement intégrée dans le déploiement de notre méthode de croisement dense appliquée aux trois visées de départ. Elle en constitue peut-être, dans le contexte éducatif ici à Marseille, le meilleur parachèvement possible. La ville détient le «record» français du plus grand nombre de REP+ (réseaux d'éducation prioritaire renforcés), au nombre de 23 (et le département des Bouches-du-Rhône, avec $33 \mathrm{REP}+$, vient en deuxième derrière celui du 
Nord). Un des arrondissements marseillais est réputé être le quartier le plus pauvre d'Europe (Péraldi, Samson, Duport, 2015). Même dans ce contexte, les enseignants et les CPE qui travaillent ici, bien que "fractions dominées de la classe dominante", appartiennent à la catégorie $n^{\circ} 3$ de l'INSEE («Cadres et professions intellectuelles supérieures »). L'écart social dans les REP+ de Marseille est donc un des plus spectaculaires qui puissent être, la reproduction y trouve un de ses terreaux les plus redoutables (Bourdieu et Passeron, 1970 ; Cnesco, 2016 ; Lahire et alii, 2019).

L'exemple sur lequel nous allons nous appuyer est celui des projets Dafip-Sfère, partenariat entre le service de la Formation et de l'innovation pédagogique du Rectorat de l'académie d'Aix-Marseille, et la Structure fédérative de recherche en éducation d'Aix-Marseille Université. En lien direct avec la Cellule recherche-développement en innovation et expérimentation pédagogique (Cardie - Rectorat) et avec l'Inspé (Amu), lui-même récemment "relocalisé » dans le $13^{\text {ème }}$ arrondissement de Marseille, dans le campus Etoile à St Jérôme, au cœur des «quartiers Nord » de la ville. Projets dont le déploiement à l'échelle de l'académie tout entière (REP+, REP, « hors REP ») vient d'être validé dans le cadre plus général du projet Ampiric (PIA3). L'impression qui prévaut souvent «sur le terrain » de ces projets est celle d'une rencontre "ordinaire » entre une demande qui émane des équipes d'enseignants et de direction, et une offre de chercheurs intéressés par cette demande - à quel prix le contexte REP+ peut-il être perçu comme ordinaire? Or, appliquée à ces projets, notre méthode aurait pour conséquence de recontextualiser systématiquement la rencontre entre la demande et l'offre, pour en densifier le dénominateur commun : l'intérêt partagé autour d'un objet d'éducation non encore construit en objet d'étude, et qui ne peut l'être que par adossement à la recherche. Mais il s'agit d'une recherche qui ne peut plus jouer le rôle " prescrit » d'éclairage, d'extériorité et de surplomb, dans ce contexte précis, sous peine de passer à côté du projet " réel », de sa dynamique, de son potentiel. Pour éviter cet écueil, une véritable clinique de la rencontre collaborative devrait avoir lieu, comme moment à part entière de la recherche, passant par une écoute active mutuelle, en vue de la co-construction effective de l'objet. Sans développer plus avant, si l'on raccorde cette relation clinique aux trois visées, on peut constater ici que l'heuristicité partagée voire co-construite avec les non-chercheurs statutaires devient synonyme de "démocratisation" de la recherche ; que la praxéologie traverse potentiellement n'importe quelle demande, obligeant les chercheurs statutaires à en tenir grand compte; que la critique, en rapport avec le contexte, ne tolère aucun académisme et aucun évitement. Car cet adossement est réflexif en ce qu'il permet d'objectiver sans concession les postures des différents acteurs de la rencontre : du côté des chercheurs statutaires, mettre à l'épreuve leurs compétences scientifiques justement dans leur capacité à croiser les trois visées; du côté des non-chercheurs statutaires, mettre à l'épreuve leur capacité à construire comme objet d'étude leur position sociale objectivement favorisée dans un contexte professionnel défavorisé. Le statut d'intellectuel professionnel redevient central, ce qui contribue à envisager l'effacement de la dichotomie chercheurs / non-chercheurs. La réflexivité ainsi construite en objet de recherche, est également un objet de formation, "naturellement» adossable à la recherche ( $c f$. parmi de très nombreux exemples, Perez-Roux, 2013). Un tel programme se situe pleinement sur le terrain de l'épistémologie «réflexive» présentée au début. Il contribue à révéler " l'épistémocentrisme scolastique " que la démarche scientifique telle que nous l'avons définie, combat jusque dans les classes, les programmes scolaires et les instructions 
officielles. «C'est ainsi que l'épistémocentrisme scolastique qu'engendre la condition scolastique est au principe d'une compréhension presque toujours erronée et déformée de la compréhension pratique. [...] Et la réflexivité, qui invite à prendre un point de vue théorique sur le point de vue théorique, est un moyen scolastique de lutte contre les dispositions scolastiques » (Mauger, 2013).

Les projets Dafip-Sfère sont potentiellement structurants de milieux formateurs. Ils peuvent contribuer à l'installation d'un dispositif de Travail dirigé délocalisé (TDD), également adossé à la recherche, notamment à l'observation in situ, en écho direct avec des points importants de la note ministérielle sur le TC évoquée dans le point précédent. "L'activité de recherche doit permettre l'acquisition de compétences en lien avec le métier d'enseignant ou de personnel d'éducation, notamment par l'observation et l'analyse des pratiques professionnelles"; outre "privilégier les expériences concrètes, en situation", «mobiliser les équipes plurielles" [pluricatégorielles]. Le professionnel, suivant les descripteurs (2015) de la CC14 (pour rappel: "S'engager dans une démarche individuelle et collective de développement professionnel », cf. point précédent), « repère, dans l'observation de la pratique du tuteur et les échanges avec les collègues, des éléments à expérimenter »; "construit des liens entre des situations professionnelles vécues et observées et des savoirs théoriques"; "conduit une analyse de son activité seul[e], en entretien et/ou en collectif de travail ; "ajuste sa pratique en conséquence "; "s'engage à suivre une formation, à mettre en œuvre une expérimentation à l'échelle de l'école, de l'établissement ", autant d'éléments qu'une recherche collaborative clinique "dense " permettrait de développer dans des proportions conséquentes. Le lien que nous venons de faire avec le TC est primordial : le REP+ serait-il le milieu formateur le plus propice pour le commun? Par exemple pour le développement (pré)professionnel ? Ce dernier rejoint la définition opératoire en éducation prioritaire qu'en donnent van Zanten et Grospiron (2001), comme capacité (peu répandue parmi les enseignants dans leur échantillon) à se décentrer de la routine professionnelle pour mieux retrouver la mission première d'enseignement. L'enseignant qui se développe professionnellement s'adosse à la recherche en prenant comme objet d'étude ce qui empêche son activité réflexive, et qui est souvent en lien avec l'impensé institutionnel. Celui-ci, entre autres, refoule l'existence de l'écart social avec ses propres élèves; par exemple, il peut ainsi objectiver, déconstruire et neutraliser une de ses manifestations les plus refoulées: le mépris intellectuel.

Tel que nous le comprenons, le projet Ampiric est le démonstrateur du changement d'échelle rendu possible par le fil conducteur croisant visées heuristique, praxéologique et critique, adossement inédit à une recherche relocalisée (en établissement formateur, et alentours) et pluricatégorielle (entre intellectuels professionnels). Ampiric permettrait d'adosser systématiquement à la recherche chaque heure de formation, initiale et continue, de tous les publics, chaque heure d'apprentissage, depuis l'école maternelle, de tous les élèves. Ce projet pourrait ainsi révolutionner l'activité intellectuelle au sens le plus large du terme, le rapport à la recherche au sens large, le rapport au savoir, «tout au long de la vie », par exemple avec un e-portfolio réflexif, scénarisé, métacognitif et partagé. Ampiric porte une tentative de révolution symbolique, le projet scientifique de toute une vie que sous-tend toute activité intellectuelle. 


\section{Conclusion : un autre rapport à la recherche} concepts, d'autres politiques, d'autres institutions et d'autres contextes. Dans le cas qui nous concerne, la situation actuelle de l'éducation à Marseille « et ailleurs... » (pour reprendre le titre d'un ouvrage fameux des sciences de l'éducation des années 1990), met durement à l'épreuve la recherche qui est censée l'objectiver, la formation des enseignants qui est censée ne pas l'ignorer, les enseignants et CPE qu'elle relègue dans les fractions dominées des classes dominantes, position sociale douloureuse s'il en est, les élèves des catégories populaires, qui confirment l'ampleur de la véracité de la sociologie de la reproduction, pour ne citer qu'elle. Face à un tel objet, un autre rapport à la recherche parait possible. La révolution scientifique pour laquelle nous plaidons n'est en fait révolutionnaire que dans son intention de retrouver un geste fondateur, qui combine, par exemple, celui des sciences de l'éducation des origines en 1967, celui de Durkheim inventant avec Mauss la sociologie générale à Bordeaux en 1890, celui de Bourdieu objectivant l'école avec Passeron dans les années 1960, puis après sans lui, tous combinant plusieurs approches scientifiques en une seule démarche, qu'il convient de nous remémorer en l'actualisant.

Leur cheminement était celui d'une sorte de radicalisation scientifique qui réévaluait tout sur son passage. Cette radicalisation a-t-elle une actualité, un avenir ? Le chemin de crête que nous avons emprunté nous semble particulièrement prometteur. La "condensation» qu'il permet, par réduction d'un volume trop important voire dispersé de pistes heuristiques, praxéologiques et critiques, et augmentation de la densité obtenue par le croisement des trois, donne naissance à un « plus petit commun dénominateur » original. Il s'agit du commun potentiel de tout travail intellectuel, des plus jeunes élèves aux plus vieux savants, celui de l'adossement réflexif à la recherche. Nous en avons décrit méthodiquement les conditions épistémologiques, théoriques, didactiques, réglementaires, empiriques, collaboratives/participatives, productrices selon nous d'un autre rapport à la recherche, développé et démocratisé par la démarche d'enquête (par exemple au cœur de l'œuvre de Dewey, actualisée par Ladage et Chevallard, par Thiévenaz). Et, plus fondamentalement encore, d'un autre rapport au savoir, qui est en fait un retour « révolutionnaire » à l'élémentaire (décrit par Lakanal pendant la Révolution française, au cœur de l'œuvre de Durkheim, cf. par exemple Sembel, 2019) ; cet inventaire historique aboutit à des conclusions sévères à l'encontre la politique scolaire menée un peu moins d'un siècle plus tard par Ferry. Ici, maintenant, tout savoir relié à l'intérêt intellectuel est fondamental, valorisant l'infinité des formes de raisonnement réflexif et de déploiement de l'esprit critique, et de ce fait vecteur d'émancipation, contre le rudimentaire sous toutes ses formes et dans tous les domaines, terreau de l'ignorance (Corbin, 2020). 


\section{BIBLIOGRAPHIE}

Abdallah-Pretceille M., 1999, L'éducation interculturelle, Paris, PUF.

Astolfi J.-P., 2008, La saveur des savoirs. Disciplines et plaisir d'apprendre, Issy-les-Moulineaux, ESF.

Boschetti A., 2014, Ismes. Du réalisme au postmodernisme, Paris, Cnrs éditions.

Bourdieu P., 2001, Esquisse d'une autosocioanalyse, Paris : Raisons d'agir.

Bourdieu P., 2003a, «L'objectivation participante », Actes de la recherche en sciences sociales, 150, 43-58.

Bourdieu P., 2003b, Science de la science et réflexivité, Paris : Raisons d'agir.

Bourdieu P., Passeron J.-C., 1970, La reproduction. Éléments pour une théorie du système d'enseignement, Paris, Minuit.

Chevallard Y., 1998, «Questions vives, savoirs moribonds : le problème curriculaire aujourd'hui ", Texte d'une communication au colloque Défendre et transformer l'école pour tous (Marseille, 1997).

Cnesco, 2016, Comment l'école amplifie-t-elle les inégalités sociales et migratoires?, 60p.

Corbin A., 2020, Terra incognita. Une histoire de l'ignorance, Paris, Albin Mchel.

Dewey J., 1993/1967, Logique. La théorie de l'enquête. Paris, PUF.

Durkheim E., 1883/1967, « Discours aux lycéens de Sens », Cahiers internationaux de sociologie.

Durkheim E., 1925/1992, L'éducation morale, Paris, PUF.

Durkheim E., 1938/1990, L'évolution pédagogique en France, Paris, PUF.

Geertz C., 1998, « La description dense », Enquête, pp. 73-105.

Heilbron J., 2018, « Sociogenèse de la théorie sociologique de Pierre Bourdieu », Zilsel, 3, pp. 105-135.

Ladage C., Chevallard Y., 2011, « Enquêter avec l'internet : études pour une didactique de l'enquête », Education \& didactique, pp. 85-116.

Lahire B., 2007, « Entretien », avec P. Costey et A. Perdoncin, Tracés, 13, pp. 235-248.

Lahire B., et alii, 2019, Enfances de classe. De l'inégalité parmi les enfants, Paris, Seuil.

Lakanal J., 1795, Rapport et projet de loi sur l'organisation des écoles primaires, présentés à la Convention nationale, au nom du Comité d'instruction publique, à la séance du 7 Brumaire.

Lebeaume J., Martinand J.-L., Reuter Y., 2007, « Contenus, didactiques, disciplines, formation. Entretien », Recherche et formation, 55, pp. 107-117.

Legardez A., Simonneaux L., 2006, L'école à l'épreuve de l'actualité - Enseigner les questions vives, Issyles-Moulineaux, ESF.

Mauger G., 2013, « Sociologie et réflexivité », pp. 145-161 dans F. Granjon, De quoi la critique est-elle le nom ?, Paris, Mare \& Martin.

Péraldi M., Duport C., Samson M., 2015, Sociologie de Marseille, Paris, La Découverte. 
Perez-Roux T., 2013, « Les enseignants en formation à l'épreuve de la réflexivité : tensions, ajustements, évolutions », pp. 115-134 dans M. Altet et alii (dir.), Former des enseignants réflexifs. Obstacles et résistances, Bruxelles, De Boeck.

Sembel N., 2003, Le travail scolaire, Paris, Nathan université.

Sembel N., 2019, «Conclusion générale : clore aussi l'ère des spécialités », pp. 419-430 dans M. Béra et N. Sembel (éds), Durkheim et la religion. Les Formes élémentaires de la vie religieuse d'hier à aujourd'hui (1912-2012), Paris : Garnier.

Thiévenaz J. 2019, Enquêter et apprendre au travail. Approcher l'expérience avec John Dewey. Dijon : Raison et Passions.

Van Zanten A., Grospiron M.-F., 2001, « Les carrières enseignantes dans les établissements difficiles : fuite, adaptation et développement professionnel », Ville-école-intégration, pp. 224-268.

Waquet F., 2015, L'ordre matériel du savoir. Comment les savants travaillent (XVIe-XXIe siècles), Paris, éd. du CNRS.

\section{RÉSUMÉS}

Cet article repose sur un parti-pris méthodologique, celui du recoupement «dense » des trois concepts d'heuristicité, de praxéologie et de critique, dans une perspective plus empirique que théorique. Leur croisement «immédiat » par une définition étymologique et intellectuelle de base est un outil de travail. Cette opération permet d'abord de construire des définitions ancrées dans une pratique, moins élaborées théoriquement mais opérationnelles empiriquement. Elle offre ensuite des perspectives nouvelles produites par ce croisement. Les résultats permettent de combiner une relecture d'auteurs de l'envergure de Durkheim et de Bourdieu avec une recomposition des éléments fondant la politique de formation des enseignants et des CPE (Conseillers principaux d'éducation) adossée à la recherche. "Stimulés » par le contexte scolaire marseillais, nous montrons qu'une révolution intellectuelle et légale est possible dans ce domaine, centrée sur un développement professionnel construit autour d'une réflexivité métacognitive et critique, construisant des apprentissages présentant les mêmes caractéristiques. Notre "méthode" pourrait permettre de croiser d'autres auteurs, d'autres concepts, d'autres politiques, d'autres institutions et d'autres contextes.

This article is based on a methodological position, that of the "thick" intersection of the three concepts of heuristicity, praxeology and criticism, from a more empirical than theoretical perspective. Their "immediate" intersection with basic etymological and intellectual definition is a working tool. This operation allows first to build definitions anchored in a practice, less elaborate theoretically but operational empirically. It then offers new perspectives produced by this crossing. The results make it possible to combine a rereading of authors of the stature of Durkheim and Bourdieu with a reorganization of the elements founding the training policy for teachers and CPEs (principal educational advisers) «backed » by research. "Stimulated " by the Marseille school context, we show that an intellectual and legal revolution is possible in this field, centered on professional development built around a metacognitive and critical reflexivity, building learning with the same characteristics. Our "method» could allow to cross other authors, other concepts, other policies, other institutions and other contexts. 
INDEX

Mots-clés : adossement, réflexivité, Durkheim, Bourdieu, activité intellectuelle

Keywords : backing, reflexivity, Durkheim, Bourdieu, intellectual activity

\section{AUTEUR}

\section{NICOLAS SEMBEL}

Aix Marseille université, ADEF, INSPÉ 